\title{
Filmography of Álex de la Iglesia
}

\section{Sources}

Data are from IMDb and Filmoteca Española (www.mcu.es). Audience and box-office details are as of I4 August 2005 but do not include video, DVD and television sales or sales abroad.

\section{As art director}

Mama (Pablo Berger, I988)

Tu novia está loca (Your Girlfriend Is Crazy) (Enrique Urbizu, I988) (poster designer)

Todo por la pasta (Enrique Urbizu, I99I)

Amor impasible (Iñaki Arteta, I992)

For Euskal Telebista

Detrás del sirimiri (Behind the Sirimiri) (1988)

Doctor Livingstone, supongo (Doctor Livingstone, I presume) (I990)

For TVE and Euskal Telebista

Inocente, Inocente (1993)

TV movies

La habitación del niño (The Baby's Room) 2005

Production company: Filmax

Películas para no dormir Series for Tele 5 (February 2006)

Leading players: Leonor Watling, Javier Gutiérrez, Sancho Gracia, Terele Pávez, Asunción Balaguer

\section{As director and co-scriptwriter}

Mirindas asesinas (Killer Mirindas) (SP) I99I, I2 mins

Producers: Jorge Oscar Guerricaechevarría, Matilde de la Iglesia 
Budget: 800,000 pesetas

Screenplay: Álex de la Iglesia

Music: various

Art direction: Álex de la Iglesia, José Luis Arrizabalaga (Arri), Arturo García Otaduy (Biaffra)

Leading players: Álex Angulo (Tubular Killer), Saturnino García (customer), Ramón Barea (bullfighting fan client), Óscar Grijalba (customer)

Enigma en el bosquecillo (SP) 2000, 5 mins

Production company:

Screenplay: Manuel Tallafe and Álex de la Iglesia

Leading players: Manuel Tallafe (Mozart), Javier Jordán (Salieri)

Released: Compressed Film Festival (www.notodofilmfest.com)

Interactive video game

Marbella antivicio (Marbella Vice) (Sp) I994

\section{Feature films}

Acción mutante (Mutant Action) (Sp) I992 [I993], 90 mins (Spain 80\% / France 20\%)

Production company: El Deseo, SA and CIBY 2000

Producers: Agustín Almodóvar (exec.), Esther García

Budget: 400,000,000 pesetas

Screenplay: Álex de la Iglesia, Jorge Guerricaechevarría

Photography: Carles Gusi

Special effects: Olivier Gleyze, Ives Domenjoud, Jean Baptiste Bonetto, Bernard André

Music: Juan Carlos Cuello, Def Con Dos and others

Art direction: José Luis Arrizabalaga (Arri), Arturo García Otaduy (Biaffra)

Editing: Pablo Blanco

Leading players: Antonio Resines (Ramón Yarritu), Álex Angulo (Álex Abadie), Frédérique Feder (Patricia Orujo), Fernando Guillén (Orujo), Enrique San Francisco (Luis María de Ostolaza), Juan Viadas (Juan Abadie), Karra Elejalde (Manitas), Saturnino García (Quimicefa), Jon Gabella (Chepa), Ramón Barea (Blind Shepherd), Alfonso Martínez (M. A.), Santiago Segura (Ezequiel)

Premiere: 3 February I993 (Madrid and Bilbao) / 5 February I993 (Barcelona) Audience: 368,180

Box office: $€ 989,807.73$

El día de la bestia (Day of the Beast) (Sp/It) I995, I03 mins (Spain 80\% / Italy 20\%) 
Production company: Iberoamericana and Sogetel (Spain) / M. G. SRL (Italy) / Canal + (Spain)

Producers: Andrés Vicente Gómez (exec.), Antonio Saura, Claudio Gaeta (delegate), Fernando de Garcillán, Leo Pescarolo, Luciano Martino (assoc.)

Budget: $300,000,000$ pesetas

Screenplay: Álex de la Iglesia, Jorge Guerricaechevarría

Photography: Flavio Martínez Labiano

Special effects: Reyes Abades

Digital effects: Daiquiri

Music: Battista Lena, Def Con Dos and others

Art direction: José Luis Arrizabalaga (Arri), Arturo García Otaduy (Biaffra)

Editing: Teresa Font

Leading players: Álex Angulo (Padre Berriartúa), Armando de Razza (Profesor

Caván), Santiago Segura (José María), Terele Pávez (Rosario), Nathalie Seseña (Mina), María de Gracia Cucinotta (Susana)

Premiere: 20 October 1995

Audience: I,4I6,7I2

Box office: $€ 4,367,321.16$

Prizes:

Spain 1996, Premios Goya (six prizes): Best Director; Best First Time Actor (Santiago Segura); Art Direction; Sound; Make-up and Hair; Special Effects

Main Prize of the Festival de Cine Fantástico 'Fantastic Arts' de GérardmerLorraine

Belgium I996, Brussels International Festival of Fantastic Film BIFFF: Méliès d'Or for Best Film

Perdita Durango (Mex/Sp) I997, I26 mins

(Spain 50\% / US 30\% / Mexico 20\%)

Production company: Lolafilms SL / Sogetel (Andrés Vicente Gómez) /Mirador SA de CV (Mexico) in association with Canal + (Spain) / Sogepaq SA / IMCINE (Mexico) Occidental Media Corp. co-production

Producers: Fernando Bovaira, Miguel Necoechea, Pablo Barbachano, Max Rosenberg (exec.), Marco Gómez (assoc.)

Budget: $\mathrm{I}, 200,000,000$ pesetas

Screenplay: Barry Gifford, Jorge Guerricaechevarría, David Trueba, Álex de la Iglesia. Based on the book $59^{\circ}$ and Raining: the Story of Perdita Durango.

Photography: Flavio Martínez Labiano

Special effects: Dan Lester

Digital effects: Daiquiri, Juan Tomicic

Music: Simon Bowswell

Art direction: José Luis Arrizabalaga (Arri), Arturo García Otaduy (Biaffra), Ana Solares, Salvador Parra, Andrew Bernard

Editing: Teresa Font 
Leading players: Rosie Perez (Perdita Durango), Javier Bardem (Romeo Dolorosa), Harley Cross (Duane), Aimee Graham (Estelle), Screamin' Jay Hawkins (Adolfo), Don Stroud (Marcello 'Mad Eyes' Santos), Demián Bichir (Catalina), Santiago Segura (Shorty Dee), Carlos Bardem (Reggie San Pedro), Alex Cox (Agent Doyle), James Gandolfini (Special Agent Woody Dumas), Harry Porter (Sheriff Tyrone 'Rip' Ford)

Premiere: 30 October 1997

Audience: 779,816

Box office: $€ 2,573,606.73$

Prizes:

Spain I998, Premios Goya (two prizes): Best Production Director; Best Makeup and Hair

Belgium I998, Brussels International Festival of Fantastic Film BIFFF

Spain 1998, Premios Fotogramas (one prize): Fotogramas de Plata for Best Actor (Javier Bardem)

Muertos de risa (Dying of Laughter) (Sp) 1998, 106 mins

Production company: Lolafilms SA / Sogetel (Andrés Vicente Gómez) with the participation of Telecinco

Producer: Luis Gutiérrez (exec.)

Budget: Information not available

Screenplay: Álex de la Iglesia, Jorge Guerricaechevarría

Photography: Flavio Martínez Labiano

Special effects: Antonio Molina, Juan Ramón Molina, Manuel F. Floriano

Digital effects: Esfera, Félix Bergés, Telson

Music: Roque Baños

Art direction: José Luis Arrizabalaga (Arri), Arturo García Otaduy (Biaffra)

Editing: Teresa Font

Leading players: Santiago Segura (Nino), José Miguel Monzón (El Gran Wyoming) (Bruno), Álex Angulo (Julián), Carla Hidalgo (Laura), Jesús Bonilla (Military member in coup d'état), María Asquerino (Nino's mother), José María Íñigo (himself), Uri Geller (himself), Antonio Resines (himself), Víctor Manuel (himself), Massiel (herself)

Premiere: I2 March 1999

Audience: $1,669,917$

Box office: $€ 6,299,097.15$

Prizes:

Spain 2000, Festival de Cine de Comedia de Peñíscola (Alicante) (one prize): Premio Calabuch for Best Actors (Santiago Segura, José Miguel Monzón [E] Gran Wyoming])

La comunidad (Common Wealth) (Sp) 2000, I07 mins

Production company: Lolafilms SA (Andrés Vicente Gómez) with the participation of Antena 3 and Vía Digital 
Producers: Juanma Pagazaurtundua (exec.), Marco Gómez (assoc.)

Budget: Information not available

Screenplay: Álex de la Iglesia, Jorge Guerricaechevarría

Photography: Kiko de la Rica

Special effects: EFE-X (Julio Navarro, Raúl Romanillos, Pau Costa)

Digital effects: Félix Bergés / Daiquiri

Music: Roque Baños

Art direction: José Luis Arrizabalaga (Arri), Arturo García Otaduy (Biaffra)

Editing: Alejandro Lázaro

Leading players: Carmen Maura (Julia), Eduardo Antuña (Charly), María Asquerino (Encarna), Jesús Bonilla (Ricardo), Marta Fernández Muro (Paquita), Paca Gabaldón (Hortensia), Ane Gabarain (Karina), Sancho Gracia (Castro), Emilio Gutiérrez Caba (Emilio), Kiti Manver (Dolores), Terele Pávez (Ramona), Roberto Perdomo (Oswaldo), Manuel Tejada (Chueca), Enrique Villén (Domínguez), Eduardo Gómez (García)

Premiere: September 2000

Audience: I,60I,86I

Box office: $€ 6,709,857 \cdot 5 \mathrm{I}$ (highest takings of 2000)

Prizes:

Spain 2000, San Sebastian International Film Festival (one prize): Concha de Plata for Best Actress (Carmen Maura); also film opening the official section Spain 2000, Premios Fotogramas (two prizes): Fotogramas de Plata for Best Actress (Carmen Maura); Best Director (Álex de la Iglesia)

Spain 200I, Premios El Mundo al Cine Vasco (four prizes): Best Director (Álex de la Iglesia); Best Script to Scriptwriters (Álex de la Iglesia, Jorge Guerricaechevarría); Best Actress (Ane Gabarain)

Spain 200I, XV Premios Goya (three prizes): Best Actress (Carmen Maura); Best Supporting Actor (Emilio Gutiérrez Cava); Best Special Effects

Spain 200I, Premios de la Unión de Actores de Madrid (Actors' Union of Madrid) (three prozes): Best Film Actress (Carmen Maura); Best Supporting Actor (Emilio Gutiérrez Caba, Terele Pávez

Premios del CEC (Círculo de Escritores Cinematográficos) (Film Scriptwriters' Club) (three prizes): Best Actress (Carmen Maura); Best Supporting Actor (Emilio Gutiérrez Caba); Best Editing

800 balas (800 Bullets) (Sp) 2002, 126 mins

Production company: Pánico Films, Álex de la Iglesia / Sogecine, with the collaboration of ICAA and Gobierno Vasco

Producers: Juanma Pagazaurtundua (exec.), María Angulo (assoc.)

Budget: 800,000,000 pesetas

Screenplay: Álex de la Iglesia, Jorge Guerricaechevarría

Photography: Flavio Labiano

Special effects: Juan Ramón Molina, Rafael Sorlozano 
Digital effects: Félix Bergés / Daiquiri

Music: Roque Baños

Art direction: José Luis Arrizabalaga (Arri), Arturo García Otaduy (Biaffra)

Editing: Alejandro Lázaro

Leading players: Sancho Gracia (Julián), Ángel de Andrés López (Cheyenne), Carmen Maura (Laura), Eusebio Poncela (Scott), Luis Castro (Carlos), Terele Pávez (Rocío), Enrique Martínez (Arrastrado), Luciano Federico (Enterrador), Ramón Barea (Don Mariano), Manuel Tallafé (Manuel), Gracia Olayo (Juli), Cesareo Estébanez (Andrés), Eduardo Gómez (Ahorcado), Eduardo Antuña (Taxista), Berta Ojeda (Ángeles), Ane Gabarain (Jacinta), Yoima Sánchez (Sonia), Alfonso Torregrosa (Jefe Policía), Juan Viadas (Monitor)

Premiere: I8 October 2002 (Madrid)

Audience: 407,337

Box office: $€ \mathrm{I}, 783,533.44$

Prizes:

Spain 2003, XVII Premios Goya (one prize): Best Special Effects (Juan Ramón Molina, Félix Bergés, Rafael Sorlozano)

Crimen ferpecto (Ferpect Crime) (Sp) 2004, I06 mins

(Spain 9०\% / Italy I०\%)

Production company: Pánico Films, Sogecine and Planet Pictures (It) with the collaboration of TVE SA, Canal+ and Euzkadi ITB

Producers: Juanma Pagazaurtundua (exec.)

Budget: $€ 4,750,000$

Screenplay: Álex de la Iglesia, Jorge Guerricaechevarría

Photography: José L. Moreno (Moti)

Special effects: Molina Efectos Especiales

Digital effects: Félix Bergés / Daiquiri

Music: Roque Baños

Art direction: José Luis Arrizabalaga (Arri), Arturo García Otaduy (Biaffra)

Editing: Alejandro Lázaro

Leading players: Guillermo Toledo (Rafael), Mónica Cervera (Lourdes), Luis Varela (Don Antonio), Enrique Villén (Inspector Campoy), Fernando Tejero (Alonso), Javier Gutiérrez (Jaime), Kira Miró (Roxanne)

Premiere: 22 October 2004 (Madrid)

Audience: 859,478

Box office: $€_{4}, 247$,I9I.24

Prizes:

France 2005 , $15^{\mathrm{e}}$ Festival du Cinéma Espagnol de Nantes (one prize): Best Actor (Guillermo Toledo) 


\section{As producer}

Play

Dos hombres sin destino (Two Men, No Destiny)

Madrid, Barcelona: Teatro Borrás (6 April to 8 May 2004)

Salamanca: Teatro Liceo (23-24 July 2004)

\section{Other films cited (in alphabetical order)}

Abre los ojos (Open Your Eyes) (Alejandro Amenábar, I997, Sp)

Aitor: la piel contra la piedra (Julio Medem [in production] 2006 or 2007 , Sp) El amor perjudica seriamente la salud (Love Can Seriously Damage Your Health) (Manuel Gómez Pereira, I997, Sp)

Angel Heart (Alan Parker, I987, US)

¡Ay Carmela! (Carlos Saura, I990, Sp)

Badlands (Terence Malick, I973, US)

Barton Fink (Joel Coen, I99I, US)

Basic Instinct (Paul Verhoeven, I99I, US)

The Believers (John Schlesinger, I987, US)

Las bicicletas son para el verano (Bicycles Are for Summer) (Jaime Chávarri, I983, Sp)

Blade Runner (Ridley Scott, I982, US)

Bonnie and Clyde (Arthur Penn, I967, US)

Carmen (Carlos Saura, i983, Sp)

Dark Star (John Carpenter, I974, US)

Después del sueño (After the Dream) (Mario Camus, I992, Sp)

Don't say a word (Gary Fleder, 200I, US)

El milagro de P. Tinto (P. Tinto's Miracle) (Javier Fesser, I998, Sp)

E1 Sur (The South) (Víctor Erice, I983, Sp)

Elmer Gantry (Richard Brooks, I960, US)

The Exorcist (William Friedkin, I973, US)

Fargo (Joel Coen, I996, US)

Fatal Attraction (Adrian Lyne, I98I, US)

Gone with the Wind (Victor Fleming [and George Cukor; Sam Wood], I939, US)

La guerra de papá (Daddy’s War) (Antonio Mercero, I977, Sp)

The Incredibles (Brad Bird, 2004, US)

Indiana Jones and the Temple of Doom (Steven Spielberg, I984, US)

Kika (Pedro Almodóvar, ı993, Fr/Sp)

La niña de tus ojos (The Girl of Your Dreams) (Fernando Trueba, I998, Sp)

La pelota vasca: la piel contra la piedra (Pelota Vasca: Skin against Stone (Julio

Medem, 2003, Sp)

La petición (Pilar Miro, I976, Sp)

Los otros (The Others) (Alejandro Amenábar, 200I, US/Sp) 
Lost Highway (David Lynch, I996, US)

Live and Let Die (Guy Hamilton, I973, US)

Mar adentro (The Sea Inside) (Alejandro Amenábar, 2004, Sp)

Misterio en la isla de los monstruos (Mystery on Monsters' Island) (Juan

Piquer, I977, Sp)

Natural Born Killers (Oliver Stone, I994, US)

Raising Arizona (Joel Coen, I987, US)

Ransom (Ron Howard, I996, US)

Sleeper (Woody Allen, I973, US)

Spaceballs (Mel Brooks, I987, US)

The King of Comedy (Martin Scorsese, I983, US)

The Secret Life of Words (La vida secretra de palabras) (Isabel Coixet, 2005)

The Silence of the Lambs (Jonathan Demme, I990, US)

Torremolinos 73 (Pablo Berger, 2004, Sp/D)

Torrente, el brazo tonto de la ley (Torrente, the Stupid Arm of the Law) (Santiago Segura, I998, Sp)

Torrente 2: Misión en Marbella (Torrente 2: Mission in Marbella) (Santiago

Segura, 200I, Sp)

Vacas (Cows) (Julio Medem, I992, Sp)

La vaquilla (The Heifer) (Luis G. Berlanga, I985, Sp)

Vera Cruz (Robert Aldrich, I953, US)

Vera, un cuento cruel (Vera, A Cruel Tale) (Josefina Molina, I973, Sp)

Viaje al centro de la tierra (Journey to the Centre of the Earth) (Juan Piquer, I98I, Sp)

Wild at Heart (David Lynch, I990, US) 